\title{
An atlas of $\mathbf{X}$ - and $\gamma$-ray pulse profiles of the Crab pulsar (PSR 0531+21): An analysis of energy and time variations ${ }^{\star}$
}

\author{
E. Massaro ${ }^{1}$, M. Feroci ${ }^{2}$, and G. Matt ${ }^{2,3}$ \\ 1 Istituto Astronomico, Univ. "La Sapienza", Unità GIFCO-CNR, Via Lancisi 29, I-00161 Roma, Italy \\ 2 Istituto di Astrofisica Spaziale, C.N.R., Via E. Fermi 21, I-00044, Frascati, Italy \\ 3 Dipartimento di Fisica, Universitá degli Studi "Roma Tre", Via della Vasca Navale 84, I-00146 Roma, Italy
}

Received July 16; accepted November 16, 1996

\begin{abstract}
We present an atlas of $\mathrm{X}$ and $\gamma$-ray pulse profiles of PSR 0531+21 (Crab pulsar) measured since 1967 . This atlas, containing 250 profiles, is useful to study in detail the behaviour with time and energy of this important source and to test models of the emission geometrical pattern. The atlas database is also accessible from Mosaic. An analysis of the energy dependence and time changes of the Crab pulsar light curve (and in particular of the $P 2 / P 1$ ratio), based on the atlas database, is presented.
\end{abstract}

Key words: pulsars: PSR $0531+21=$ crab pulsar atlas - X-rays: stars - gamma rays: observations

\section{Introduction}

The pulsar phenomenon was discovered about 30 years ago (Hewish et al. 1968). It was soon clear that this emission must be produced by high energy particles streaming in the magnetosphere of a rotating neutron star (Gold 1968; Pacini 1968). Several models and calculations were performed afterwards to explain the observational results. Despite these theoretical efforts many problems are still open and, in particular, that of the location of the emitting regions in the magnetosphere. Phenomenological models, based on pulse profiles in different bands of the electromagnetic spectrum, have been proposed (see, for instance Rankin 1991; Ulmer 1994), but a fully satisfactory scenario has not been established yet. Under this respect the Crab pulsar, PSR 0531+21 (formerly indicated as NP 0532), is a unique object because of its high luminosity and proximity. In fact, it can be observed across the entire electromagnetic spectrum - from radio frequencies to $\gamma$ rays - and, furthermore, it is the only pulsar for which

* Table 2 is available only in electronic form at the CDS via anonymous ftp to cdsarc.u-strasbg.fr (130.79.128.5) or via http://cdsweb.u-strasbg.fr/Abstract.html high energy data covering a rather long time interval are available.

In recent times the problem of long term changes of the $\gamma$-ray pulse profile of PSR $0531+21$ has been debated: Kanbach (1990) suggested a possible periodicity of the $P 2 / P 1$ ratio (i.e. the ratio between the relative intensities of the two major peaks, which are well evident in all spectral bands) at energies greater than about $50 \mathrm{MeV}$. After the Compton-GRO observations, the significance of this finding has been discussed in some other papers (Ulmer 1994; Ramanamurthy et al. 1995), but no conclusive result has been reached. Other attempts to search for $P 2 / P 1$ changes in the X-ray and low energy $\gamma$-ray band have also been carried out (Ulmer 1994; Nagase 1994; Massaro et al. 1995), but these analyses are complicated by the strong dependence of the ratio $P 2 / P 1$ on the energy.

We decided, therefore, to collect all the pulse profiles of PSR 0531+21, from soft X-rays to medium-high energy $\gamma$ rays, available in the literature since the early observations. We then prepared an ATLAS (Massaro et al. 1996) and organized a computer database which is available on the Mosaic home page of the Istituto Astronomico of the Università "La Sapienza" (Roma) at http://astrm2.rm.astro.it.

In the next sections we describe the database and use it to investigate some problems concerning the structure of the pulse profile such as the dependence on energy and time of the ratio $P 2 / P 1$, the shape of $P 1$, usually considered the most stable feature, and the $P 1-P 2$ phase separation.

\section{The database}

The present version of the database contains 250 pulse profiles of PSR $0531+21$, observed from 1967 to 1993 and published in the main astrophysical and astronomical journals and conference proceedings. Furthermore, a few profiles reported in Ph.D. theses or unpublished are also included. 
The profiles are divided in five groups according to their energy ranges. The code of these groups, their energy intervals, the numbers of profiles and of used original papers are given in Table 1. Within each group the profiles are generally ordered by observation epoch and, for the same epoch, by increasing average energy. Each profile, in addition to the group code, is identified by two numbers: the first specifies either the observation epoch or the experiment, the second distinguishes the different energy ranges and/or phase binnings.

All the profiles in the Atlas and their relevant parameters are listed in Table 2. The identification code of each profile is reported in Col. 1, in Col. 2 there is the observation epoch, in cols. 3 and 4 the number of phase bins and the nominal energy ranges respectively, in Col. 5 the phase bin of the $P 1$ centre (with the approximation of half a bin) in the original figure and, finally, in Col. 6 the paper code.

In order to make easier their use, we have for all the profiles: taken the phase of the $P 1$ centre equal to 0 ; subtracted an off-pulse level, estimated by computing the mean in the phase interval $(0.55-0.77)$, the most narrow of those used in the literature; normalized to unity the maximum of $P 1$.

The accuracy of the profiles is good: digitization errors are usually much smaller than the statistical ones, with the only possible exception for those derived from very small or poor quality original figures.

The database is organized as follows. All the data and service files are written in ASCII to make simpler their examination, editing and transfer. General information on the content and use of the database and Atlas are given in the file CRPATL.INF and are updated when new data are available or when new consulting facilities are available. The list of all profiles can be found in the file CRPATL.IND, which is reproduced in Table 2. Another service file CRPATL.REF, containing all the bibliographical references, listed in alphabetical order according to the reference code reported in CRPATL.IND.

The data files are two for each profile; both files are named by the profile code but have different extensions: for example, SX01-1.CRP and SX01-1.NOR. The files with the CRP extension have records of 80 characters. The first two records are divided in four fields of 20 characters each, containing the file name, the reference code, the observation date or period, the energy interval and some other useful information. The third record contains the bin number and the off-pulse level evaluated in the original paper (a value equal to zero means this level is not indicated in the paper). The following records contain the values and the corresponding uncertainties of the signal in each phase bin: four couples of floating point numbers are written in each record, with the exception of the last one in which only the data necessary to complete the profile are written. When possible the scale of these quantities is the same as in the original paper, in a few case, however, they are given in arbitrary units. The files with the NOR extension contain the normalized profiles reported in the paper version of the Atlas. They do not have header and each record contains the phase of the bin centre, the normalized signal and its uncertainty.

The three service files are already accessible via Mosaic at the address given above; in a short time all the profiles and data files will also be available.

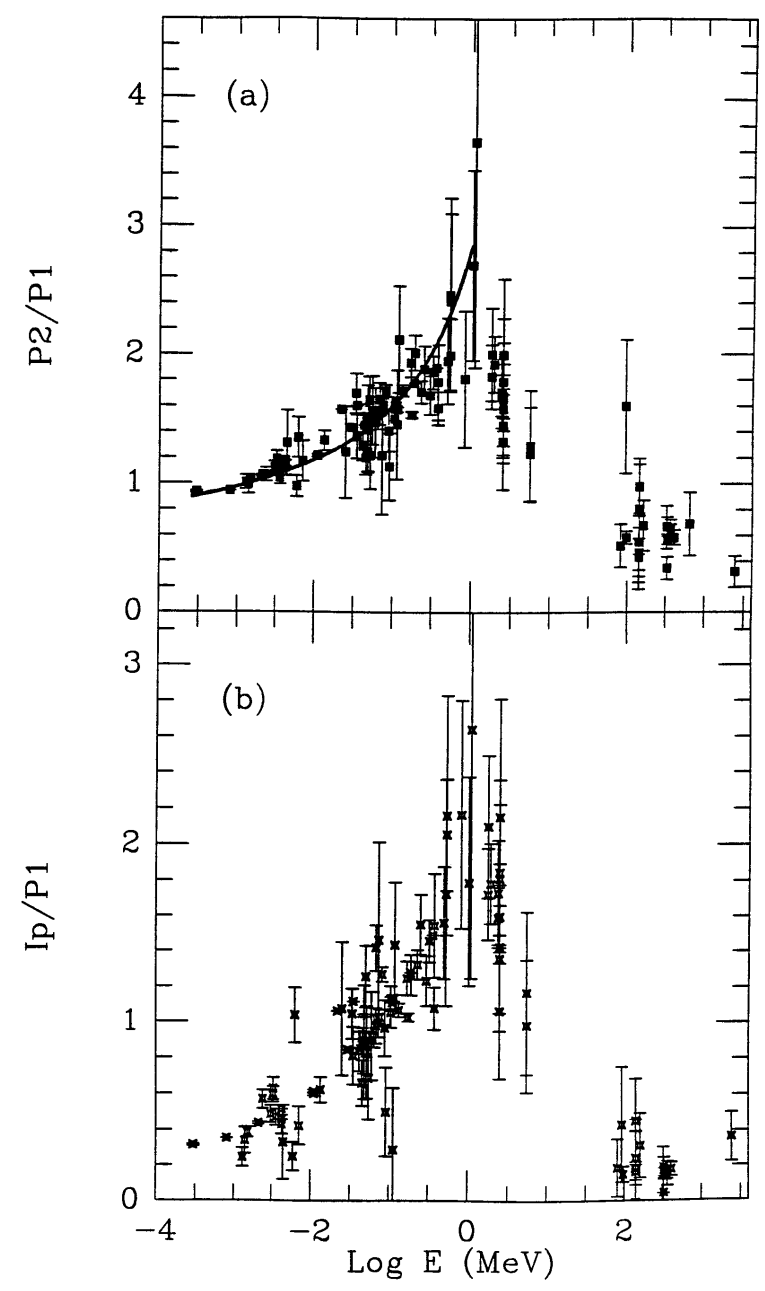

Fig. 1. The energy dependence of $P 2 / P 1$ and of $I p / P 1$, panels a) and b) respectively. The solid line in the panel a) is the plot of the Eq. (1)

\section{Study of the profile shape}

In this Sect. we present, as an example of how this database can be used, the results on a few debated problems concerning the changes of the profile structures with energy and time. 
Table 1. Profile groups in the Atlas

\begin{tabular}{lrll}
\hline Group & Energy range & Number of profiles & Number of ref. \\
\hline SX & $0.1-2.0 \mathrm{keV}$ & 9 & 8 \\
MX & $2.0-20 . \mathrm{keV}$ & 35 & 18 \\
HX & $20 .-200 . \mathrm{keV}$ & 69 & 32 \\
LG & $0.2-30 . \mathrm{MeV}$ & 81 & 28 \\
HG & $>30 . \mathrm{MeV}$ & 56 & 18 \\
\hline
\end{tabular}

\subsection{The energy dependence of the $P 2 / P 1$ ratio}

A relevant point in the study of the $P 2 / P 1$ ratio is the definition of the phase boundaries of the various features, and particularly that between the Interpeak (or "bridge") region $I p$ and $P 2$. Different values of this phase, usually in the interval $0.27-0.32$, can be found in the literature. We choose the value of 0.3 because the intensity levels at the $P 1-I p$ and the $I p-P 2$ separations in the soft $\mathrm{X}$-rays are nearly equal. The phase boundaries are: $P 1(0.95-0.05)$, $I p(0.05-0.30), P 2(0.30-0.47), B K G(0.55-0.85)$.

The results, obtained using only those profiles with a good enough statistics, are shown in Figs. 1a,b: the values of $P 2 / P 1$ (panel a) and of $I p / P 1$ (panel b) are plotted as a function of the effective detection energy, defined as the average of the nominal photon energy interval (in $\mathrm{MeV}$ ), weighted with a power law with a photon index 2.2. $P 2 / P 1$ increases from 0.95 at $0.3 \mathrm{keV}$ to $\sim 3.5$ at about $1 \mathrm{MeV}$, while at higher energies it decreases down to values close to 0.35 in the $\mathrm{GeV}$ range. A very similar behaviour is apparent in the $I p / P 1$ values, indicating a common origin of these changes.

The increasing trend of $P 2 / P 1$ for photon energies $E<1 \mathrm{MeV}$ can be described by the simple formula

$(P 2 / P 1)=2.5(1-\log (E / 1 \mathrm{MeV}))^{-1}+0.35$.

Even if it was not derived by means of a best fit, we think that it could be useful in several cases, when this dependence must be taken into account: for example, to compare the time variations $P 2 / P 1$ at different energies.

\subsection{The time dependence of the $P 2 / P 1$ ratio}

The major problem in the study of the time variations of $P 2 / P 1$ is that the observations are not well distributed in time and therefore the sampling of the various possible time scales is far from a regular coverage. In the softmedium X-rays no evidence of significant variations has been found so far: in particular the two GINGA observations (profiles MX 15-1, MX 15-2 and MX $17-1$, MX $17-2$ in Table 2), 4.5 years apart, give the same $P 2 / P 1$ and $I p / P 1$ values. Also the Einstein (SX $05-2)$ and the ROSAT (SX 07-1) profiles are practically coincident after about 12 years.

Significant variations of the peaks' intensities have been found at energies higher than $30 \mathrm{MeV}$. These were discovered in the $C O S$ - $B$ data, which showed a strong decrease of $P 2$, which in September-October 1979 become undetectable (Clear et al. 1987). Kanbach (1990) suggested that $P 2 / P 1$ changes according to a sinusoidal law, with a period of about 13.5 years, an amplitude of about 0.5 and the maximum epoch at 1972.3 . However, recently Ramanamurthy et al. (1995), after including all the nine EGRET observations, did not found any firm evidence for this periodicity, even if variations are evident. We searched for the sinusoidal behaviour in the hard $\mathrm{X}$ and low energy $\gamma$ rays, where the $P 2 / P 1$ ratio is higher.

We analyzed separately the data in the two energy intervals $20-100 \mathrm{keV}$ and $100 \mathrm{keV}-1 \mathrm{MeV}$ : the plots of $P 2 / P 1$ against time are given in Figs. 2a,b, respectively. No significant periodicity is apparent: a sine function, with the same phase of the maximum given above, does not give acceptable fits. An amplitude of 0.37 is actually found for the highest energy range; however, using Eq. (1) to scale the measured values to the intermediate energy of $0.3 \mathrm{MeV}$ (Fig. 2c), the much smaller amplitude value of 0.077 is obtained, suggesting that the previous result can be an artifact of combining measures at different energies.

Variations of the $P 2 / P 1$ ratio are nevertheless evident in the hard X-rays and in the low/high energy $\gamma$ rays. In particular, in the $>30 \mathrm{MeV}$ range, both COS$\mathrm{B}$ and EGRET detected significant changes as already said (Clear et al. 1987; Ramanamurthy et al. 1995). With our choice of the phase boundaries, $P 2 / P 1$ changes from $0.97 \pm 0.20$ (HG $13-6$ ) in 1975 to $0.26 \pm 0.11$ (HG $15-3$ ) in 1979 and from $0.60 \pm 0.06$ (HG $21-2$ ) to $0.395 \pm 0.090$ (HG $23-1)$ in about one month of 1991 . Both changes are due to an evident decrease of the $P 2$ intensity.

We found significant changes of the $P 2 / P 1$ ratio at lower energies. The values of $1.20 \pm 0.13$ and $1.52 \pm 0.08$ were found for the profiles HX $10-1(23-103 \mathrm{keV})$ in 1976 and HX 18-1 (30-80 keV) in 1981. An even greater difference is found between the profiles HX 17-3 (110$190 \mathrm{keV})$ and HX $24-3(110-220 \mathrm{keV})$ : the former has a $P 2 / P 1$ ratio equal to $1.94 \pm 0.11$, while the latter gives $1.535 \pm 0.025$, In this case, however, rather than a change in the content of $P 2$, such a difference should be attributed to a change in $P 1$. In the low-energy $\gamma$-ray profiles no difference more significant than 1.5 standard deviations has been found. 


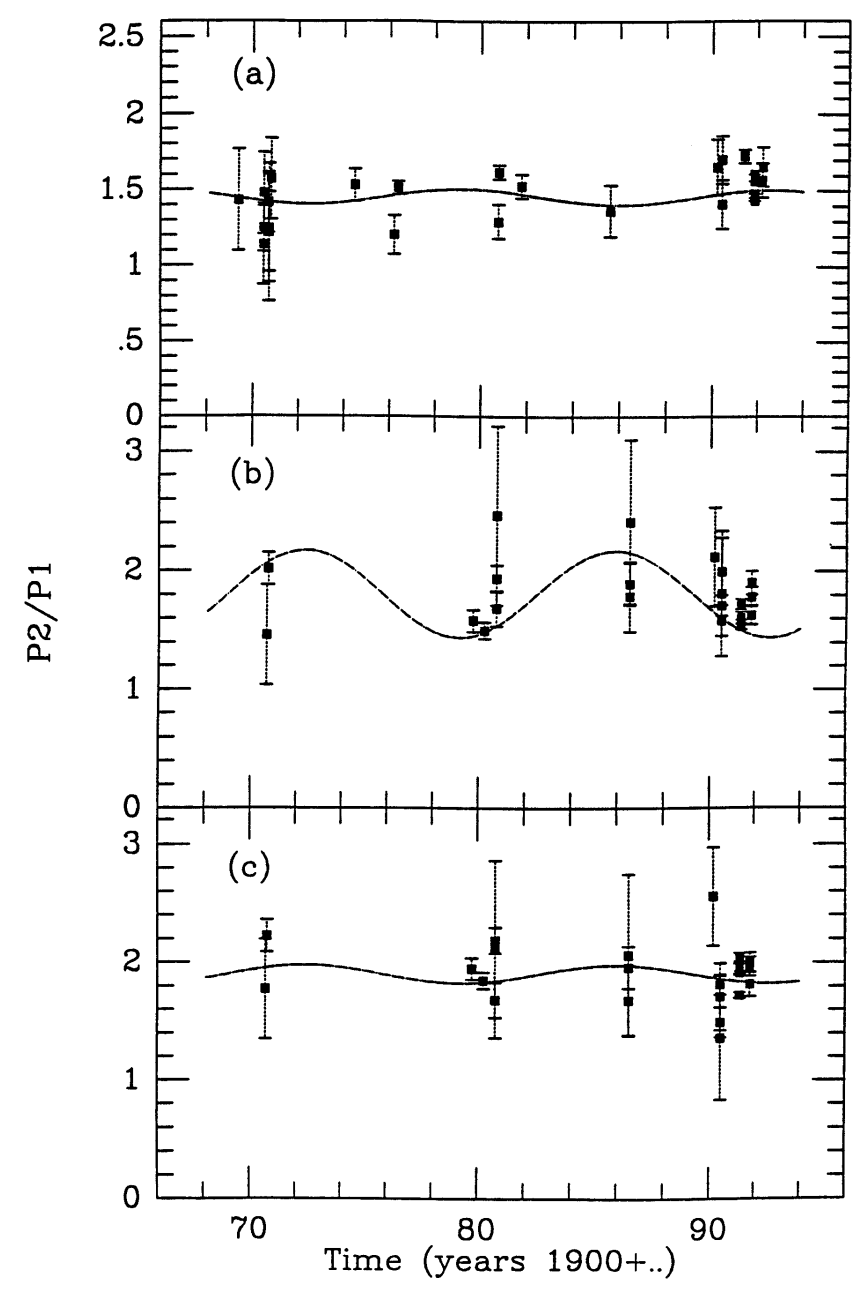

Fig. 2. The time dependendece of $P 2 / P 1$ for the profiles in the energy range $20-100 \mathrm{keV}$ a) and $100-1000 \mathrm{keV}$ without energy scaling b) and after the scaling according to Eq. (1) c). The dashed lines represent the best fit sinusoids with the same phase of Ramanamurthy et al. (1995)

\subsection{The shape of $P 1$}

We used the high statistics ROSAT profile (SX $07-1$ ) as a template for the higher energies and searched for a simple analytical formula able to fit the shape of $P 1$. We choose first a symmetric generalized bell shaped profile

$y=A \exp \left(-\left|\phi-\phi_{0}\right|^{\alpha} / b\right)$

where $\phi$ and $\phi_{0}$ are the phase and the central phase of the peak, respectively, and $y$ is the normalized bin content. $\alpha$ resulted very close to unity and $\phi_{0}$ to zero, but the fit was not satisfactory because the shape is not symmetric. We then modified Eq. (2) introducing different values of $b$ for the leading and trailing sides and fixing $\alpha=1$ and $\phi_{0}=0$. Even if the reduced $\chi^{2}$ is 1.8 (34 d.o.f.), the result, shown in Fig. 3a, can be considered satisfactory to achieve a global and simple description of the $P 1$ shape. The values of the two $e$-folding constants are $b_{-}=0.03$ and $b_{+}=0.022$ for the leading and trailing sides, respectively. The same effect is also apparent at optical frequencies: the detailed profile of $P 1$ measured with HST shows a trailing side significantly steeper than the leading one (Percival et al. 1993). Afterwards, we verified whether the same function is a good description of the other profiles apart from the normalization. Significant deviations were found for the profiles in the hard $\mathrm{X}$ and low $\gamma$ rays. Two examples are shown in Figs. 3b and 3c: one is the profile HX $18-1(30-80 \mathrm{keV})$ and the other one is LG $12-7$ at energies greater than $150 \mathrm{keV}$. The disagreement is mainly in the trailing side, where significant excess counts from phase 0.01 onwards are apparent. At variance, at energies greater than $50 \mathrm{MeV}$ (EGRET 100 bin data reported by Thompson et al. 1993 - Fig. 3d), there is no evidence for a different shape of the ROSAT one.

\subsection{P1-P2 phase separation}

Ramanamurthy (1994) suggested a monotonic decrease with energy of the intra peak separations like for the Vela pulsar. The Atlas permits to search for regular changes over more than six orders of magnitude in energy.

The skewness of $P 1$ is small enough at all energies to estimate its maximum phase as the centroid of a symmetric distribution. On the other hand, $P 2$ is fairly symmetric only in the low energy $\mathrm{X}$ and medium-high energy $\gamma$ rays, but it is very skew in the hard $\mathrm{X}$ and low energy $\gamma$ rays; the use of a symmetric algorithm would give a bias toward smaller phase values. We therefore estimated the phase of the $P 2$ maximum either by computing the barycentre of a few (typically five) phase bins, for all the energies at which it can be considered symmetric, or the phase of the bin with the highest content in the other cases. An uncertainty of half a bin was always taken.

The phase separation varies between $0.384 \pm 0.010$ and $0.410 \pm 0.010$, corresponding to two HG profiles. No significant evidence for a decreasing trend was found and the data are only slightly scattered with respect to the mean value 0.399 over the entire $(0.1 \mathrm{keV}-100 \mathrm{MeV})$ interval. All these peak separations are smaller that those given by Percival et al. (1993) at optical and UV frequencies, equal to $0.4167 \pm 0.0004$ and $0.4139 \pm 0.005$, respectively. Elder optical data (Warner et al. 1969; Cocke \& Ferguson 1974) give phase separation values in the interval $0.41-$ 0.42 , confirming this difference. We can conclude, therefore, that a decrease of the $P 1-P 2$ phase distance of about 0.015 between the optical and X-ray frequencies is real, while no significant change is appreciable at energies greater than about $0.5 \mathrm{keV}$.

\section{Conclusion}

PSR 0531+21 (Crab pulsar) is undoubtedly the best studied among isolated pulsars, but several problems concerning the origin of the pulsed radiation and the location of 


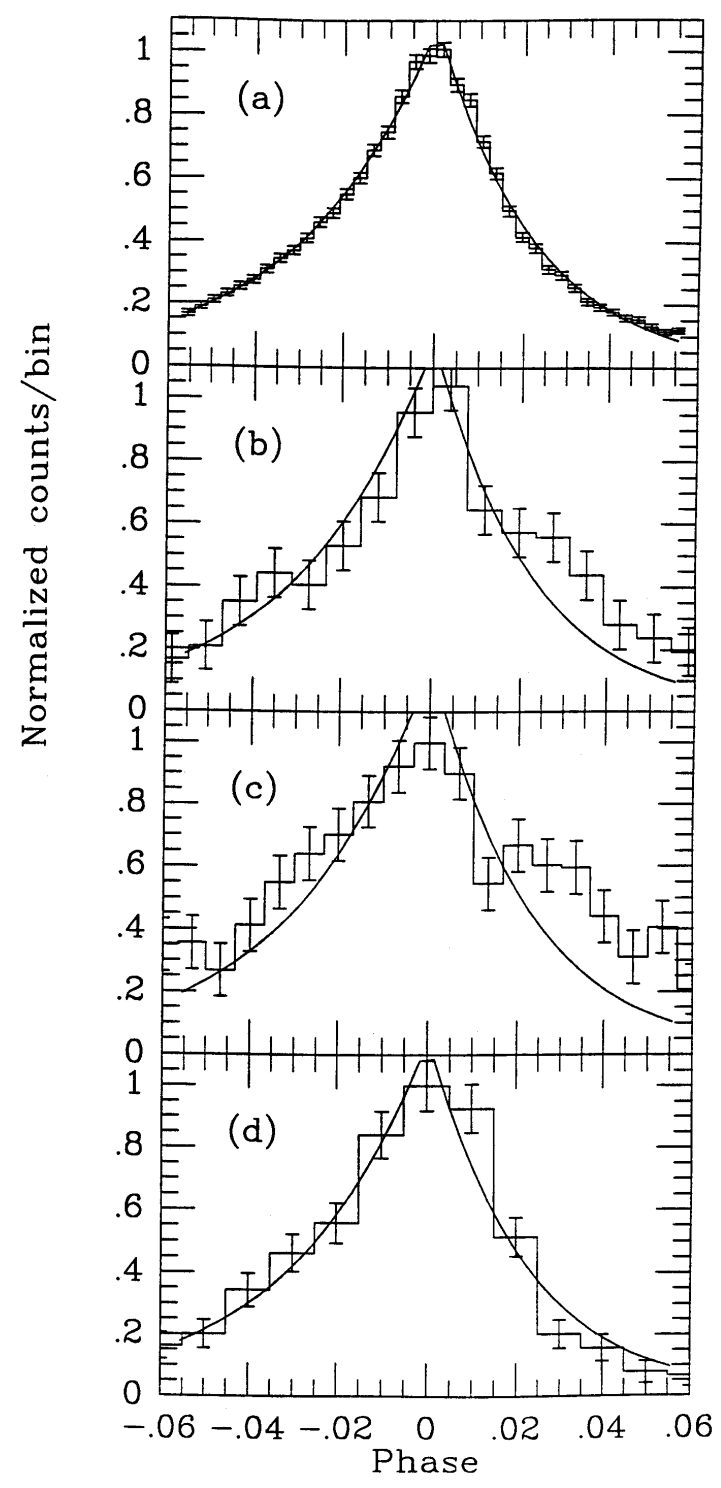

Fig. 3. The shape of $P 1$ at four different energies: $0.1-2.5 \mathrm{keV}$ (SX 07-1) a) used as a template profile to evaluate parameters of the double exponential function, $30-80 \mathrm{keV}$ (HX $18-1$ ) b), $0.15-2 \mathrm{MeV}(\mathrm{LG} 12-7)$ c) and $>50 \mathrm{MeV}(\mathrm{HG} 21-1)$. Notice that the analytical shape is not able to represent hard $\mathrm{X}$ and low energy $\gamma$-ray data, while it is well suited to the high energy $\gamma$-ray profile

the emitting regions within the magnetosphere are still open. To achieve more information on them we have realized a computer accessible database containing an Atlas as complete as possible of $\mathrm{X}$ and $\gamma$-ray pulse profiles appeared in the literature since its discovery. This Atlas could be not complete because some data may have not been published yet. The Crab remnant and the pulsar, in fact, are frequently observed by various astrophysical space instruments (it is often used as a reference source) but not always these data are presented in the literature. We ask then all the scientists to help us in improving this database, which we hope can be useful to many people, by adding to the Atlas either the original data files of already published data in order to avoid errors due to the figure digitization, or results which they do not intend to publish elsewhere. Any other contribution to complete the collection of the past observation of the Crab pulsar, and to continue the data collection with new data, is welcome. As a further comment, this Atlas is also useful to trace back the history of the early $\mathrm{X}$ and $\gamma$-ray experiments which had often the Crab as the primary target and which are now scarcely quoted in the literature, despite some of them provided excellent data.

We used the Atlas profiles to investigate some subjects which have been recently discussed in the recent literature. We do not find evidence for the 13.5 years periodicity of the $P 2 / P 1$ ratio in the $\mathrm{X}$ and low energy $\gamma$ rays. We confirmed the time stability of this quantity in the soft and medium X-ray range, while, at energies greater than about $30 \mathrm{keV}$, some changes are apparent, but their occurrence and time scales are still practically unknown. We obtained also the most complete picture of the energy dependence of the $P 2 / P 1$ and $I p / P 1$ ratios, which both reach their maximum around $1 \mathrm{MeV}$. A further point studied here is the shape of $P 1$ : a comparison of the Atlas profiles has shown that it is not the same at all energies, but the right side is characterized by an additional component in the hard $\mathrm{X}$ and low-energy $\gamma$ rays. This result can be taken as an indication that the traditional feature separation of the Crab pulse profile into $P 1, I p$ and $P 2$ could not be appropriate. Conversely, one could adopt a different choice separating the profile in an inner region, namely that between the maxima of the peaks, and in an outer region. Such a model can be physically based on a conical emission pattern having the vertex close to one polar cap of the neutron star. The photons of inner region would then be originated closer to the star magnetic pole than the others and therefore have a different spectral distribution with a break at about $1 \mathrm{MeV}$. The overall stability of this pattern, in energy and time, is indicated by the observed phase separation between the edges of the two sections.

Acknowledgements. We are grateful to the FIGARO Collaboration for the permission to include in the Atlas some unpublished profiles. We are also indebted to W. Becker who gave us the data file of the ROSAT data. We are particularly grateful to M. Litterio for the help in organizing the $W W W$ access to the computer database. This work has been carried out with the financial support of the Consiglio Nazionale delle Ricerche (GIFCO research contract).

\section{References}

Clear J., et al., 1987, A\&A 174, 85

Cocke W.J., Ferguson D.C., 1974, ApJ 194, 725

Gold T., 1968, Nat 218, 731

Hewish A., et al., 1968, Nat 217, 709

Kanbach G., 1990, in "The EGRET Science Symposium", Fichtel C.E. et al. (eds.) NASA CP-3071, p. 101 
Massaro E., Matt G., Feroci M., 1995, Proc. 24th ICRC, OG 3.1.8, 2, 199

Massaro E., Matt G., Feroci M., 1996, Istituto Astronomico, Report 9601

Nagase F., Zylstra G., Mihara T., 1994, in "New horizon of X-ray Astronomy", Makino F. and Ohashi T. (eds.) p. 57 Pacini F., 1968, Nat 219, 145

Percival J.W., et al., 1993, ApJ 407, 276

Ramanamurthy P.V., 1994, A\&A 284, L13
Ramanamurthy P.V., et al., 1995, ApJ 450, 791

Rankin J.M., $\quad$ 1991, in "Neutron Stars: Theory and Observation", Ventura J. and Pines E. (eds.). Kluwer Acad. Publ., p. 349

Thompson D.J., et al., 1993, in "Isolated Pulsars", van Riper K.A., Epstein R. and Ho C. (eds.). Cambridge Univ. Press, p. 230

Ulmer M.P., 1994, ApJS 92, 789

Warner B., et al., 1969, Nat 222, 233 